\title{
Exploiting Multi-Channel Diversity in Spectrum-Agile Networks
}

\author{
Alexander W. Min and Kang G. Shin \\ Real-Time Computing Laboratory, EECS Department \\ The University of Michigan, Ann Arbor, MI 48109-2121 \\ \{alexmin,kgshin\}@eecs.umich.edu
}

\begin{abstract}
Recently, spectrum-agile (SA) networks have been recognized as a viable solution to the spectrum-scarcity problem in wireless communications. In SA networks, secondary (unlicensed) users are allowed to opportunistically utilize idle licensed spectrum bands, thus improving spectrum utilization efficiency and accommodating more users and applications. We take a twostep approach to the problem of maximizing the throughput of an SA network. The first step is to determine a subset of "candidate" channels that a secondary device will consider for its channel-switching. The candidate channels are selected based on their estimated utilization. We then propose channel-aware switching to determine when and where to switch to, among the candidate channels. Wireless channels are assumed to experience independent Rayleigh fading, and modeled with a finite-state Markov channel (FSMC) model. Our evaluation results show that the proposed channel-aware switching scheme significantly outperforms the traditional forced-switching scheme in terms of average throughput.
\end{abstract}

\section{INTRODUCTION}

Spectrum-agility (cognitive radio) [1] is a revolutionary technology with great potential for solving the spectrumscarcity problem that is beginning to surface as the number of wireless applications and users increases rapidly. This problem is, in fact, rooted in the conventional static spectrum allocation policy where only a primary (or licensed) user (PU) is allowed to operate on its designated spectrum band, and this results in a severe under-utilization of wireless spectrum bands as reported in [2], [3]. This problem can be alleviated by spectrumagility, which allows a secondary (or unlicensed) user (SU) to opportunistically utilize the unused licensed spectrum bands and thus enables flexible and efficient use of the entire radio spectrum.

For opportunistic use of spectrum bands, an SU must be aware of its dynamically-changing local wireless environment and be able to quickly adapt to it. Awareness of the wireless environment can be accomplished via a channel-sensing mechanism. For instance, an SU periodically senses its operating channel to detect PUs so that it can vacate the channel immediately when a PU reclaims the channel. Moreover, an SU can also benefit from sensing by obtaining useful channel statistics such as primary channel utilization and ON/OFF distributions. Therefore, in this paper, the purpose of sensing is not limited to primary detection but also includes estimation and prediction of various aspects of local wireless environments.

Besides channel sensing, an SU should be able to adaptively switch to the best available channel, to improve the system performance. The "best" channel here means the one that maximizes the throughput of a secondary network and it is determined based on two key channel characteristics: (i) availability of the channel, which depends on PUs' channel utilization, and (ii) quality of the channel, which fluctuates over time and location due to various unpredictable phenomena such as fading, shadowing, and interferences. The best available channel can be found by our proposed channelaware switching scheme, which exploits the characteristics of multiple channels in SA networks.

To be effective, the channel-aware switching must address the following MAC-layer sensing and switching issues:

- How many or which channels must be sensed in order to maximize spectrum access time?

- How does an SU determine the best channel that maximizes throughput?

We first discuss how to determine the candidate channels for channel sensing by mathematically analyzing the tradeoff between the sensing overhead and the probability of finding a spectrum opportunity. Then, we propose an algorithm for making channel-switching decisions by considering both the channel availability and instantaneous channel conditions. Note that our key idea is to maximize throughput by utilizing the best channel while minimizing the corresponding overheads.

There have been only a few publications addressing the channel-switching problem in a multi-channel wireless environment. The authors of [4] proposed a Multi-channel Opportunistic Auto Rate (MOAR) protocol which finds the optimal number of channels to skip in search for a betterquality channel. The authors of [5] proposed a On-Demand Channel switching (ODC) to minimize the switching delay by keeping a node in its channel as long as the channel condition is acceptable. Although these approaches propose ideas for channel-switching in a multi-channel environment, they focus on IEEE 802.11 WLANs and do not deal with the unique challenges (e.g., primary activity) posed by SA networks. Moreover, they do not consider different channel characteristics in channel-switching decisions.

\section{System Models AND Assumptions}

There are three basic assumptions behind our proposed scheme. First, a licensed wireless spectrum is assumed to consist of a set $\mathcal{N}$ of non-overlapping channels, each with fixed channel bandwidth $B$. Second, PUs can access any channel, while SUs can access any channel only when the channel is found to be idle via channel sensing. A channel is said to be idle or available if there is no PU activity on that channel. Third, a secondary network consists of a pair of communicating SUs, and each SU is equipped with a single half-duplex transceiver capable of switching channels dynamically. 


\section{A. Primary User's Channel Usage Model}

We model the presence or absence of PU's signal on each channel as a continuous-time, alternating ON/OFF Markov renewal process (MRP) $\{S(t): t \geq 0\}$, as shown in Fig. 1. The validation of this model for the presence of PU's signal can be found in [6], [7]. The ON state indicates that a PU is currently using the channel, while the OFF state implies no PU activity on that channel which is called a spectral opportunity or a spectrum hole. The sojourn times of ON and OFF states are assumed to be i.i.d. random variables $X_{k}$ and $Y_{k}, k \in \mathbb{N}$, with the distribution functions $F_{X}$ and $F_{Y}$, respectively. The ON/OFF distributions can be estimated by an SU, based on the sequence of channel-sensing results (i.e., ON/OFF pattern) [8]. A transition from OFF to ON state incurs a renewal and the duration of the $k^{\text {th }}$ renewal period is denoted as $Z_{k}=X_{k}+Y_{k}$.

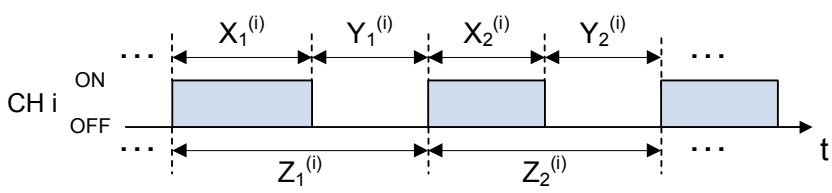

Fig. 1. Alternating MRP model for PU's channel utilization

\section{B. Channel or Spectrum Sensing}

In general, each SU must periodically sense its current channel to detect the presence of PU's signal. In our proposed channel-switching scheme, an SU periodically scans not only his current channel but also other candidate channels with sensing period $T_{s}$. This way, whenever an SU is interrupted (e.g., due to detection of an incumbent), it can promptly switch channel based on the recently-acquired channel information. For example, an SU can switch to the least-utilized channel by exploiting the channel characteristics (e.g., channel utilization and ON/OFF distributions) obtained by periodic channel sensing.

\section{Wireless Channel Model}

Wireless channels are assumed to experience independent Rayleigh fading (i.e., channels are separated by more than a coherent bandwidth). A Rayleigh fading channel can be accurately modeled as a finite-state Markov channel (FSMC) in a slow fading environment [9], [10]. Thus, the FSMC model with a state space $S=\left\{s_{1}, s_{2}, \ldots, s_{M}\right\}$ is used to describe the signal-to-noise ratio (SNR) fluctuations of each channel, as shown in Fig. 2. In the figure, the $\left\{C_{i}\right\}_{i=0}^{M}$ and $\left\{L_{i}\right\}_{i=1}^{M}$ represent the SNR thresholds of the model for each state and corresponding fading regions, respectively. The state-transition probabilities $\left\{p_{i, j}\right\}, i, j \in S$ are estimated by the method used in [9].

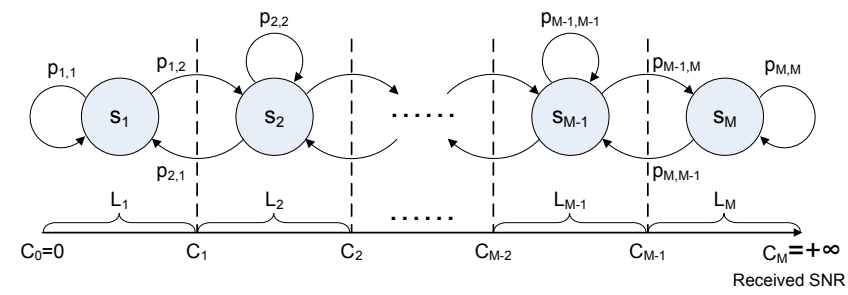

Fig. 2. Finite-state Markov channel (FSMC) model for Rayleigh fading

\section{Adaptive Modulation}

To exploit the time-varying nature of a wireless environment, an SU needs to provide high flexibility in terms of modulation and coding. Hence, we assume that an SU supports eight different transmission rates by employing the physicallayer (PHY) modulation schemes defined in the IEEE 802.11a PHY standard [11]. ${ }^{1}$ We assume that an SU adopts different PHY modes for each channel state (i.e., fading region) of the FSMC model and determines the SNR thresholds $\left\{C_{i}\right\}_{i=0}^{M}$ to guarantee the target bit-error-rate (BER), which can be adjusted to meet the required QoS of various wireless applications.

\section{Channel-Aware Switching in SA Networks}

Channel-switching is indispensable for SUs to access the licensed spectrum opportunistically, since they are not allocated dedicated channels and must immediately vacate their current channel as soon as a PU returns. In this section, two channelswitching schemes are introduced: (i) forced-switching and (ii) channel-aware switching. In forced-switching, an SU monitors only the current channel and switches to a randomly selected channel upon detection of PUs, as shown in Fig. 3. In this case, an SU makes a channel-switching decision without considering the underlying channel characteristics. Therefore, substantial performance degradation may unavoidable, while having a minimal sensing and switching overheads.

In our proposed channel-aware switching, an SU proactively switches to the best channel to maximize the expected throughput. Thus, an SU initiates the channel-switching procedure when either (i) a PU returns to the current channel or (ii) the channel-switching period $T_{s w}$ expires, whichever occurs first, as illustrated in Fig. 4. Moreover, instead of randomly selecting a channel, an SU considers all of different channel characteristics-such as center frequency, fading level, and primary activity-for channel-switching decision to fully exploit the diversity of multiple channels. For instance, an SU estimates the PU's channel utilization using the periodic channel sensing results (i.e., a sequence of binary ON/OFF patterns). In addition, an SU measures the channel SNR at each channel-switching decision time to estimate the temporal channel condition.

Clearly, the channel-aware switching can improve the throughput performance at the cost of increased sensing and switching overheads. Therefore, our channel-aware switching scheme exploits the tradeoff between the operational overheads and the resultant throughput enhancement by taking a twostep procedure for channel-switching. Our scheme first decides a set of candidate channels which maximize the spectrum accessibility and then derive a channel-switching decision rule to maximize throughput.

\section{A. Selection of Candidate Channels}

Here the candidate channel selection algorithm is introduced, which exploits the tradeoff between the spectrum opportunity and sensing overhead. Note that, enlarging the set of candidate channels increases the probability of discovering a spectrum opportunity (i.e., idle channel), but incurs more interruptions to the $\mathrm{SU}$, preventing his opportunistic use of

\footnotetext{
${ }^{1}$ The current IEEE 802.22 draft PHY supports QPSK,16-QAM, and 64 QAM with convolutional coding schemes of rate 1/2,3/4, and 2/3 [12].
} 


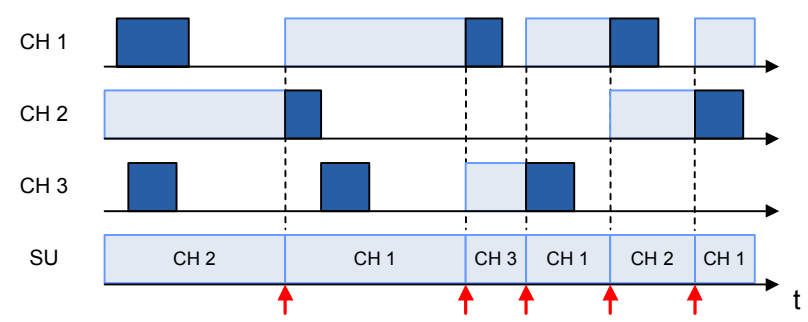

Fig. 3. An example of forced-switching

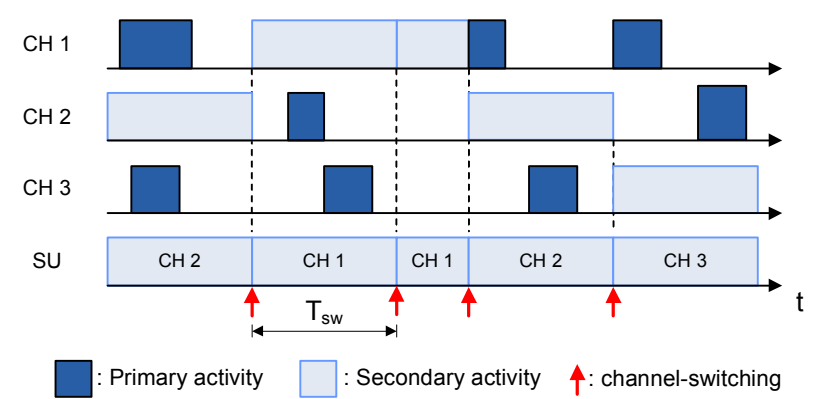

Fig. 4. An example of channel-aware switching

available channels. Therefore, our objective is to optimize the tradeoff in selecting the set of candidate channels by maximizing the average spectrum access time, which can be an indirect measure of throughput performance.

First, given a candidate channel set $\Omega$, our algorithm calculates an average fraction of time when there is at least one idle channel for an SU to utilize. That is,

$$
\begin{aligned}
\xi(\Omega) & =1-\mathrm{P}(\text { no idle channel in } \Omega) \\
& =1-\prod_{i \in \Omega} \hat{u}_{i},
\end{aligned}
$$

where $\hat{u}_{i}$ is the estimated channel utilization on candidate channel $i$. Note that, in practice, an SU can accurately estimate the channel utilization $\hat{u}_{i}$ using the sample mean estimator based on the sequence of binary ON/OFF patterns collected from periodic channel sensing [8].

Then, it calculates the spectrum accessibility $\varphi$ as:

$$
\varphi(\Omega)=\xi(\Omega)\left(1-O_{s c}\right),
$$

where $O_{s c}=\max \left\{|\Omega| \times \frac{\Delta T}{T_{s}}, 1\right\}$ denotes the total fraction of time spent on channel sensing where $\Delta T, T_{s}$, and $|\Omega|$ are the sensing duration, sensing period, and number of candidate channels, respectively.

Finally, the algorithm determines the optimal set of candidate channels $\Omega^{*}$ by solving the following optimization problem:

$$
\begin{gathered}
\text { Find } \Omega^{*}=\arg \max _{\Omega} \varphi(\Omega) \\
\text { subject to } \quad \Omega \subset \mathcal{N},
\end{gathered}
$$

where $\mathcal{N}$ is the set of all channels. Note that the candidate channels should be updated periodically since PU activity on each channel is a time-varying process.

\section{B. Channel-Aware Switching}

Given a set of candidate channels, an SU must find the best channel that maximizes the throughput $\mathcal{G}$ until the next channel-switching time. For this end, an SU requires the following channel information: (i) current channel condition (i.e., SNR), (ii) expected channel holding time, and (iii) FSMC model. Note that it is assumed that the channel-switching delay $\Delta d$ is fixed, and the channel-switching period $T_{s w}$ is a design parameter and the effect of its choice will be evaluated using simulation.

1) Channel SNR Measurement: At each channel-switching decision time, an SU first sequentially scans the candidate channels to identify idle channels, and then measures the channel SNR of idle channels. Let $\Gamma=\left\{\gamma_{i}\right\}_{i \subset \Theta}$ denote a set of measured SNR values where $\Theta \subset \Omega$ is a set of idle channels at channel-switching decision time.

2) Channel-Holding Time: The channel-holding time $\left(T_{h}\right)$ is defined as the time duration over which an SU can utilize an idle channel before getting interrupted. Obviously, frequent channel-switching (i.e., small $T_{h}$ ) can degrade the throughput performance significantly. Therefore, the channel-holding time should be considered in channel-switching decision.

According to the renewal theory [13], the distribution of channel-holding time $F_{T_{h}}$ converges as follows, provided the channel is in the OFF state:

$$
\lim _{t \rightarrow \infty} F_{T_{h}}(t)=\int_{0}^{t} \frac{1-F_{Y}(s)}{E[Y]} \mathrm{d} s,
$$

where $F_{X}$ and $F_{y}$ are the distributions of channel $\mathrm{ON}$ and OFF states, respectively as shown in Fig. 1.

The PU activity (i.e., ON/OFF state transitions) can be described as a two-state (i.e., ON and OFF) Markov chain. Let $t r_{i j}(t)$ denotes the probability that the channel is in state $j$ at time $t$, provided the channel started in state $i$ at time 0 $(i, j \in\{0,1\})$. That is,

$$
t r_{i j}(t)=\mathrm{P}(S(t)=j \mid S(0)=i) .
$$

Assuming that the channel ON and OFF periods are both exponentially distributed with p.d.f. $f_{X}(t)=\lambda_{X} e^{-\lambda_{X} t}$ and $f_{Y}(t)=\lambda_{Y} e^{-\lambda_{Y} t}(t \geq 0)$, we have [14]:

$$
\begin{aligned}
& t r_{00}(t)=\frac{\lambda_{X}}{\lambda_{X}+\lambda_{Y}}+\frac{\lambda_{Y}}{\lambda_{X}+\lambda_{Y}} e^{-\left(\lambda_{X}+\lambda_{Y}\right) t} \\
& \operatorname{tr}_{01}(t)=\frac{\lambda_{Y}}{\lambda_{X}+\lambda_{Y}}-\frac{\lambda_{Y}}{\lambda_{X}+\lambda_{Y}} e^{-\left(\lambda_{X}+\lambda_{Y}\right) t} .
\end{aligned}
$$

Since the channel-switching is followed by a channelsensing, which is performed at a discrete-time interval (i.e., integer multiples of $T_{s}$ ), the channel-holding time $T_{h}$ can take one of the values $\left\{T_{s}, 2 T_{s}, \ldots, n_{s} T_{s}\right\}$ where $n_{s}=\left\lceil\frac{T_{s w}}{T_{s}}\right\rceil$ is the number of channel sensing within a channel-switching decision period $\left(T_{s w}\right)$. Therefore, the p.m.f. of channel-holding time can be expressed as:

$$
\begin{aligned}
& \mathrm{P}\left(T_{h}=k T_{s}\right) \\
& =\left\{\begin{array}{lll}
\prod_{j=1}^{k-1} t r_{00}\left(j T_{s}\right) t r_{01}\left(k T_{s}\right) & \text { if } & k=1,2, \ldots,\left(n_{s}-1\right) \\
1-\sum_{j=1}^{n_{s}} \operatorname{tr}_{00}\left(j T_{s}\right) & \text { if } \quad k=n_{s} .
\end{array}\right.
\end{aligned}
$$




$$
E\left[\mathcal{G}^{(i)} \mid \gamma_{i} \in L_{m}\right]=\sum_{k=1}^{n_{s}-1} \frac{v_{m}(k l)}{k T_{s}+\Delta d} \operatorname{tr}_{01}\left(k T_{s}\right) \prod_{j=0}^{k-1} \operatorname{tr}_{00}\left(j T_{s}\right)+\frac{v_{m}\left(n_{s} l\right)}{n_{s} T_{s}+\Delta d} \prod_{j=0}^{n_{s}} \operatorname{tr}_{00}\left(j T_{s}\right)
$$

$$
E\left[\mathcal{G}^{(i)} \mid \gamma_{i} \in L_{m}\right]=\sum_{k=1}^{n_{s}} \frac{v_{m}(k l)}{k T_{s}+\Delta d}(1-p)^{k-1} p+\frac{v_{m}\left(n_{s} l\right)}{n_{s} T_{s}+\Delta d}(1-p)^{n_{s}}
$$

3) Expected Throughput Estimation: We assume that the channel fading level is constant for a single time slot of duration $\tau$. The size of $\tau$ can be up to a few packet transmission times, depending on how fast (or slow) the fading is. We can then calculate the expected throughput for each idle candidate channel by accumulating the expected number of data bits transmitted in each time slot for the channel holding time, which is not known a priori.

A reward vector is defined as $\mathbf{r}=\left[r_{1} r_{2} \cdots r_{M}\right]^{T}$ where $r_{i}$ is the reward (i.e., total number of data bits transmitted during $\tau$ ) in state $i$. Assume that there are $n$ time slots until the next channel-switching decision time. Then, the total expected data bits transmitted until the next switching-decision time, given the initial state $i$, can be calculated as:

$$
v_{i}(n)=r_{i}+\sum_{j=1}^{M} p_{i, j} v_{j}(n-1)
$$

where $p_{i, j}$ is the state-transition probability of the FSMC model. This can be expressed in vector form as:

$$
\mathbf{v}(n)=\mathbf{r}+\mathbf{P} \mathbf{v}(n-1) .
$$

By replacing Eq. (12) iteratively, and assuming that $\mathbf{v}(0)=\mathbf{r}$, we can obtain an explicit equation for $\mathbf{v}(n)$ as:

$$
\begin{aligned}
\mathbf{v}(n) & =\mathbf{r}+\mathbf{P r}+\mathbf{P}^{2} \mathbf{r}+\cdots+\mathbf{P}^{n} \mathbf{r} \\
& =\sum_{k=1}^{n} \mathbf{P}^{k} \mathbf{r} \quad k \in \mathbb{N} .
\end{aligned}
$$

In general, the number of time slots, $n$, within a switching period $T_{s w}$ is very large, since $T_{s w} \gg \tau$. Therefore, instead of directly calculating the exact value of $\mathbf{v}(n)$, we can approximate it by introducing a relative gain vector $\mathbf{w}=\left[w_{1} w_{2} \cdots w_{M}\right]^{T}$, where $w_{i}$ denotes the relative advantage of starting at state $i$ over the other states, as follows [14]:

$$
\mathbf{v}(n) \approx \mathbf{w}+n g \mathbf{e}+\beta \mathbf{e},
$$

where $g=\sum_{i=1}^{M} \pi_{i} r_{i}$ is the average expected reward in the steady-state and $\mathbf{e}$ is a unit vector.

By substituting Eq. (12) into Eq. (14), we get:

$$
\mathbf{v}(n)=\mathbf{r}+\mathbf{P}(\mathbf{w}+(n-1) g \mathbf{e}) .
$$

Then, based on Eqs. (14) and (15), we can derive the following equation:

$$
w_{i}+g=r_{i}+\sum_{j=1}^{M} p_{i, j} w_{j}
$$

Since $p_{i, j}=0$ if $|i-j|>1 \forall i, j$, we have:

$$
w_{i}+g=r_{i}+p_{i, i-1} w_{i-1}+p_{i, i} w_{i}+p_{i, i+1} w_{i+1} \text {. }
$$

TABLE I

THE CHANNEL PARAMETERS IN THE SIMULATION

\begin{tabular}{|c|c|c|c|c|c|c|c|}
\hline & $\mathrm{CH} 1$ & $\mathrm{CH} 2$ & $\mathrm{CH} 3$ & $\mathrm{CH} 4$ & $\mathrm{CH} 5$ & $\mathrm{CH} 6$ & $\mathrm{CH} 7$ \\
\hline \hline freq $(\mathrm{MHz})$ & 300 & 350 & 400 & 450 & 500 & 550 & 600 \\
\hline $\mathrm{E}[\mathrm{X}](\mathrm{s})$ & 1.5 & 1.0 & 1.5 & 1.0 & 1.5 & 3.0 & 2.0 \\
\hline $\mathrm{E}[\mathrm{Y}](\mathrm{s})$ & 2.0 & 4.5 & 5.5 & 2.0 & 8.0 & 3.5 & 1.5 \\
\hline Util $(\%)$ & 42.9 & 18.2 & 21.4 & 33.3 & 15.8 & 46.1 & 57.1 \\
\hline $\bar{\gamma}(\mathrm{dB})$ & 22.02 & 21.26 & 20.44 & 19.52 & 18.50 & 17.34 & 16.0 \\
\hline
\end{tabular}

TABLE II

SENSING AND SWITCHING OPERATION PARAMETERS

\begin{tabular}{|c|c|c|c|}
\hline \multicolumn{2}{|l|}{ Sensing } & \multicolumn{2}{l|}{ Switching } \\
\hline \hline$T_{s}$ & $200 \mathrm{~ms}$ & $T_{s w}$ & $50 \sim 800 \mathrm{~ms}$ \\
\hline$\Delta \mathrm{T}$ & $2 \mathrm{~ms}$ & $\Delta \mathrm{d}$ & $1 \mathrm{~ms}$ \\
\hline
\end{tabular}

Thus, by substituting the state-transition probabilities of the FSMC model into Eq. (17), we have:

$$
g-r_{i}=\frac{N_{C_{i-1}}}{R_{i}}\left(w_{i-1}-w_{i}\right)+\frac{N_{C_{i}}}{R_{i}}\left(w_{i+1}-w_{i}\right),
$$

where $N_{C_{i}}$ is the expected downward transition rate across the threshold $C_{i}$ and $R_{i}$ is the average packet transmission rate per second in state $s_{i}$, respectively [9]. Therefore, the relative gain vector can be calculated based on the FSMC model parameters.

By adding a constraint, $\sum_{i=1}^{M} \pi_{i} w_{i}=0$, meaning that an average relative gain in steady-state is 0 , we have [14]:

$$
\mathbf{v}(n) \approx \mathbf{w}+n g \mathbf{e}+\mathbf{P}^{n}(\mathbf{r}-\mathbf{w})
$$

4) Channel-Switching Decision Rule: At each channelswitching decision time, the expected throughput of an idle candidate channel $i \in \Theta$ can be computed as shown in Eq. (4), where $\gamma_{i}$ is the measured channel SNR and $l=\frac{T_{s}}{\tau}$ is the number of time slots within a sensing period $T_{s}$. In case of exponential $\mathrm{ON} / \mathrm{OFF}$ distributions, the ON/OFF channel state-transition probabilities do not depend on the channel-switching time (i.e., memoryless property). Thus, the expected throughput can be expressed as shown in Eq. (5) with $p=\operatorname{tr}_{01}\left(T_{s}\right)$.

Finally, the channel-switching strategy for maximum throughput is to switch to channel $i^{*}$ such that:

$$
i^{*}=\arg \max _{i \in \Theta} E\left[\mathcal{G}^{(i)} \mid \gamma_{i}\right]
$$

\section{PERformance EVAluation}

We now evaluate the effectiveness of the proposed channelaware switching via simulation with MATLAB. We will discuss a few important observations due to the space limitation. 


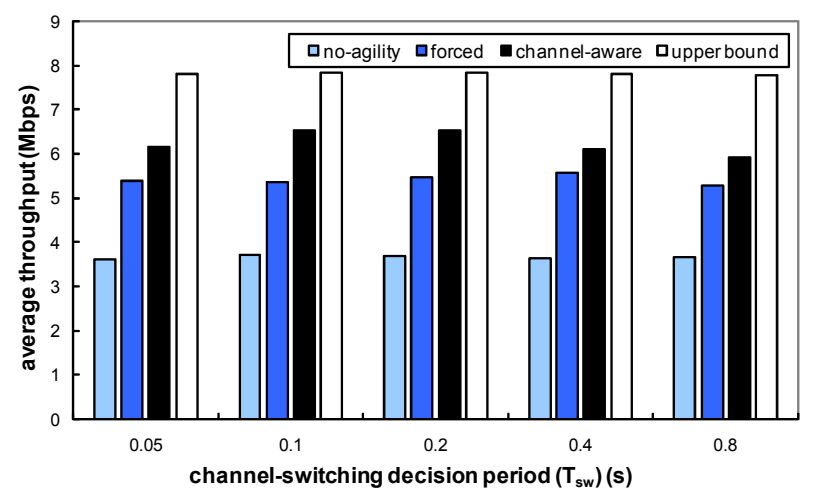

Fig. 5. Effect of channel-switching decision period

\section{A. Simulation Setup}

For a wireless environment, we assume seven channels with different characteristics as shown in Table I. We summarized the channel sensing and switching related parameters in Table II. In the simulation, we model the SNR fluctuations of each channel with an 8-state FSMC model. For each Markov state, we employ different modulation schemes (i.e., BPSK, QPSK, 16-QAM, and 64-QAM) with various rate-adaptation convolutional codes for forward error correction (FEC), and determine the SNR thresholds of the FSMC model based on the target BER of $10^{-4}$.

We evaluate the performance of the following switching schemes: (i) no spectrum-agility, (ii) forced-switching, (iii) channel-aware switching, and (iv) perfect-switching. In the no-spectrum-agility scheme, we assume that an SU randomly selects any channel with an equal probability and utilizes it only when the channel is idle. There is no channel-switching in this case. For perfect-switching scheme, we assume that an SU always utilizes the best channel without any sensing or switching overheads. Therefore, we use the achieved throughput of this scheme as a performance upper bound. We also assume that an SU transmits packets in a greedy mode (i.e., its data queue is always full, so an idle channel can be fully utilized).

\section{B. Simulation Results}

1) Effect of Channel-Switching Decision Period $\left(T_{s w}\right)$ : Fig. 5 shows that the proposed channel-aware switching scheme outperforms the forced-switching in terms of average throughput under all the simulated scenarios. As shown in Fig. 5, when the switching-decision period is relatively small (i.e., $T_{s w}<200 \mathrm{~ms}$ ), the performance degrades due to increased sensing and switching overheads. On the contrary, the performance also degrades when the switching-decision period is relatively large due to lack of agility in channel-switching.

2) Effect of Maximum Doppler Shift $\left(f_{m}\right)$ : In Fig. 6, the performance of the channel-aware switching scheme decreases as the Doppler shift increases (i.e., the mobile's speed increases) because the channel condition changes faster with a high Doppler shift. When the Doppler shift is close to zero (i.e., $0.001 \mathrm{~Hz}$ ), our scheme achieves $91 \%$ of the performance upper bound, while the forced-switching scheme achieves only $69 \%$ of the upper bound.

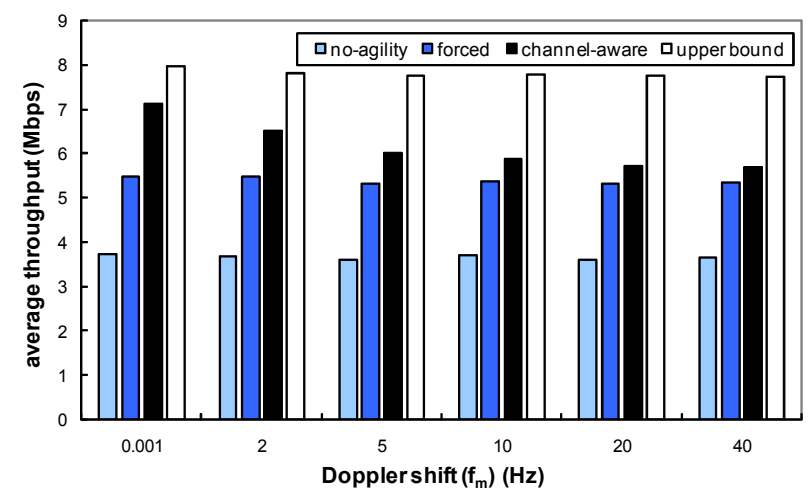

Fig. 6. Effect of maximum Doppler shift

\section{CONCLUSION}

For efficient use of spectrum resources in a time-varying SA wireless environment, we proposed a channel-aware switching scheme, which maximizes throughput by exploiting multiple channels in SA networks. We assumed a Rayleigh fading for wireless channels and modeled them as a finite-state Markov channel (FSMC) model. For our channel-aware switching scheme, we developed a candidate channel selection algorithm to maximize the spectrum accessibility and then derived the channel-switching decision rule to determine the best channel to switch to. The simulation results show that our scheme outperforms the forced-switching due mainly to its ability to analyze the channel characteristics and exploit the dynamic nature of the wireless environment.

\section{REFERENCES}

[1] S. Haykin, "Cognitive Radio: Brain-Empowered Wireless Communications," IEEE Journal on Selected Areas in Communications, vol. 23, no. 2, pp. 201-220, Feb 2005.

[2] FCC, "Spectrum Policy Task Force," Rep. ET Docket No. 02-135, Nov 2002.

[3] M. McHenry, "NSF Spectrum Occupancy Measurements Project Summary," Shared Spectrum Company Report, Aug 2005. [online]. Available: http://www.sharedspectrum.com/?section=measurements

[4] V. Kanodia, A. Sabharwal, and E. Knightly, "MOAR: A Multi-channel Opportunistic Auto-rate Media Access Protocol for Ad Hoc Networks," in Proc. IEEE BROADNETS, Oct 2004.

[5] P. Porwal and M. Papadopouli, "On-demand channel switching for multi-channel wireless MAC protocols," TR04-024 Report, University of North Carolina, 2004.

[6] A. Motamedi and A. Bahai, "MAC Protocol Design for Spectrumagile Wireless Networks: Stochastic Control Approach," in Proc. IEEE DySpan, Apr 2007.

[7] S. Geirhofer, L. Tong, and B.M. Sadler, "Dynamic Spectrum Access in WLAN Channels: Empirical Model and Its Stochastic Analysis," in Proc. ACM TAPAS, Aug 2006.

[8] H. Kim and K.G. Shin, "Efficient Discovery of Spectrum Opportunities with MAC-Layer Sensing in Cognitive Radio Networks," to appear in IEEE Transactions on Mobile Computing.

[9] H.S. Wang and N. Moayeri, "Finite-State Markov Channel - A Useful Model for Radio Communication Channels," IEEE Transactions on Vehicular Technology, vol. 44, no. 1, pp. 163-171, Feb 1995.

[10] H.S. Wang and P.-C. Chang, "On Verifying the First-Order Markovian Assumption for a Rayleigh Fading Channel Model," IEEE Transactions on Vehicular Technology, vol. 45, no. 2, pp. 353-357, May 1996.

[11] IEEE 802.11a, Part 11: Wireless LAN Medium Access Control (MAC) and Physical Layer (PHY) Specifications: High-speed Physical Layer in the $5 \mathrm{GHz}$ Band. Supplement to IEEE 802.11 Standard, June 2003.

[12] C. Cordeiro, K. Challapali, and D. Birru, "IEEE 802.22 : An Introduction to the First Wireless Standard based on Cognitive Radio," IEEE Journal of Communications, vol. 1, no. 1, Apr 2006.

[13] D.R. Cox, Renewal Theory, Butler \& Tanner Ltd, London 1967.

[14] R.G. Gallager, Discrete Stochastic Processes, Kluwer Academic Publishers, 1996. 